\title{
Is $5 \%$ dextrose prolotherapy effective for radicular low back pain?
}

\author{
Özlem Köroğlu ${ }^{1}$, Aydan Örsçelik ${ }^{2}$, Özlem Karasimav ${ }^{2}$, Yasin Demir ${ }^{1}$, İlker Solmaz ${ }^{3}$ \\ (1) University of Health Sciences, Gaziler Physical Treatment and Rehabilitation Training and Research Hospital, \\ Department of Physical Medicine and Rehabilitation, Ankara, Turkey
}

(2) University of Health Sciences, Gulhane Training and Research Hospital, Department of Sports Medicine, Ankara, Turkey

(3) University of Health Sciences, Gulhane Training and Research Hospital, Department of Traditional and Complemantary Medicine, Ankara, Turkey

Date submitted:

Jun 13, 2019

Date accepted:

Jul 29, 2019

Online publication date:

September 15, 2019

\section{Corresponding Author:}

Özlem Köroğlu

University of Health Sciences,

Gaziler Physical Treatment

and Rehabilitation Training and

Research Hospital, Department

of Physical Medicine and

Rehabilitation, Ankara, Turkey

drozlemkoroglu@gmail.com

Keywords: Prolotherapy, dextrose, radicular low back pain, lumbar disc herniation.

\begin{abstract}
Aims:Prolotherapy is a regenerative treatment that induces the local inflammation response and has been used clinically for many musculoskeletal system disorders. Dextrose is the most common solution that is used for prolotherapy. Dextrose prolotherapy can be used as a complementary treatment for various musculoskeletal system disorders including low back pain. The aim of our trial is to assess the potential effectiveness of $5 \%$ dextrose injection for radicular low back pain resulting from lumbar disc herniation.
\end{abstract}

Methods:We evaluated a total of 40 patients treated with $5 \%$ dextrose prolotherapy for radicular low back pain for one year. Of these patients, 20 patients were treated with only prolotherapy. The other 20 patients were treated with prolotheraphy and physical therapy. 5\% dextrose solution is used for the treatment and the solution being injected to iliolumbar and transverse ligament insertion levels and at the facet level. We used Visual Analog Scale (VAS) $10 \mathrm{~cm}$, Oswestry Disability Index (ODI) and Short Form-36 (SF-36) for the evaluation of pain, mobility, quality of life and functional status of the patients.

Results:A significant reduction in pain was reported in all patients in both groups. The pain and disability scores significantly improved both in two groups at the 3, 12 and 52-week follow-up with no significant difference between the groups.

Conclusions:Patients with radicular low back pain resulting from disc herniation can be treated effectively by using $5 \%$ dextrose prolotheraphy.

\section{Introduction}

Prolotherapy is a regenerative treatment that induces growth of the connective tissue due to the local inflammation response and it has been used clinically for multiple musculoskeletal system disorders. Prolotherapy has targeted mainly on entheses as a origin of pain and this treatment technique is guided by anatomic education and precise physical examination, especially palpation. Prolotherapy includes the injection of irritant solutions to the joint spaces and at soft tissue connections in order to reduce pain and enhance function in painful musculoskeletal system disorders. The main aim of prolotherapy is to treat ligamentous laxity for solving the related musculoskeletal and arthritic pathologies. Many kinds of solutions have been used for prolotherapy treatment but dextrose is the most common irritant solution for prolotherapy injections. Dextrose is considered to be an ideal proliferant because it has a normal component of blood chemistry, can be injected safely into multiple areas and in large volumes and it is water soluble. Still the mechanism of effect is not well understood and it is probably multifactorial (1,2 and 3). Although we do not know the effect mechanisms of dextrose clearly, we know that hyperosmolar dextrose dehydrates cells at the injection site until they rupture by creating a large osmotic gradient and starts an inflammation affecting the cells. This process attracts the granulocytes and macrophages. Inflammation plays a very important role in repairment pathway since inflammatory cytokines regulate the cellular environment that controls the advance of other repair changes. Therefore, we can say that inflammation is the best way to develop an effective repair. When non-steroidal anti-inflammatory drug (NSAID) treatment is used, the inflammatory reaction is seriously blocked for a prolonged period of time and this causes an incomplete tissue healing which may result more risk of increasing scar formation and injury recurrence. Also prolotherapy induces the production of the growth factors and cytokines that cause a better tissue healing. Dextrose stimulates regeneration of the joint cartilage and also supports the maturation of the collagen fibers and fibrous structures (1, 2 and 4)

Dextrose prolotherapy aims to repair the ligamenteous injuries which are the common reason for musculoskeletal pain. The injury of ligaments causes the underlying basis of disc herniation because the ligaments hold the disk in its place. The 
main reason of low back pain is mechanic pathologies especially overuse, ligament sprains, muscle problems or disc herniation (3). Radicular low back pain resulting from lumbar disc herniation is a common health problem that conservative treatments can be ineffective. Dextrose prolotherapy is a cheap and effective complementary treatment method to decrease pain for various musculoskeletal system disorders including low back pain. In the literature for extraarticular injections dextrose with concentrations ranging from $12.5 \%$ to $25 \%$ is widely used but inflammation with high concentration of dextrose can not be tolerated by some patients. Dextrose concentrations higher than $10 \%$ induces inflammatory mechanisms, while concentrations less than $10 \%$ do not cause inflammation but start proliferation $(2,3)$. So in our routine practice we use $5 \%$ dextrose for periarticular prolotheraphy treatments and we increase the concentration of dextrose if it is necessary for every individual patient. An adult fibroblast culture study showed that $5 \%$ dextrose solution increases gene expression in angiogenetic and apoptotic factors (5). The present study examines the outcome of the potential effectiveness of $5 \%$ dextrose injection for radicular low back pain due to lumbar disc herniation.

\section{Methods}

The patients treated with prolotherapy for radicular low back pain were evaluated by chart review between December 2016 and April 2018. This study has a retrospective design. The written informed approval was received from all patients. The patients who had chronic radicular low back pain for more than 3 months and diagnosis of lumbar disc herniation based on the clinical and magnetic resonance imaging findings were included in this study. The research was approved by bioethics and research committee (reference number: 18/276). The exclusion criteria included: age < 18 years old, neurological disorders, malignancy, inflammation, spinal fracture, heart failure, severe respiratory disease, renal or hepatic failure, pregnancy, rheumatic diseases, prior spinal injections, prior spinal surgery and patient's refusal. We obtain all details about the patients' medical background in the baseline visit.

The pain level of patients was measured using the visual analogue scale (VAS) $10 \mathrm{~cm}$. Oswestry Disability Index (ODI) and Short Form- 36 (SF-36) were used for the evaluation of mobility, functional condition and quality of life of the patients. The SF36 is a patient-reported questionnaire that covers eight health domains: bodily pain, physical functioning, role limitations due to personal or emotional problems, physical health problems, social functioning, emotional well-being, general health perceptions and energy/fatigue. Oswestry Disability Index is a tool to assess functional disability that is gold standard for low back pain. Prolotherapy injections contained three injections at four weeks periods.

The follow-up of patients was done at 3, 12 and 52 weeks following the first prolotherapy injection. $5 \%$ dextrose solution was used for the treatment and the solution being injected to iliolumbar and transverse ligament insertion levels and at the facet level. Patients were asked to stop NSAID at least one week before the each injection. We also asked patients not to take NSAID after the injections, only paracetamol was allowed for pain management. A total of 40 patients enrolled; 20 patients were selected from the patients whom followed with just prolotherapy treatment and 20 were selected from the patients whom treated with prolotheraphy and physical therapy. We selected the patients to treatment groups in order to pro- vide no significant difference in the continuance of symptoms, pretreatment medication or baseline VAS, Oswestry and SF-36 between the groups. The ODI, VAS and SF-36 were used in each assessment. During the patient selection, we recognized that we had offered physical therapy to all 40 patients according to their pain level but 20 of them accepted the physical therapy.

After baseline clinical assessment, prolotherapy injections were performed by the same physician (IS) using a $23 \mathrm{G}$ $0.6 \times 60 \mathrm{~mm}$ or $0.6 \times 80 \mathrm{~mm}$ injector according to patient adipose tissue. A total of three injections were applied to the patients. All patients' physical therapy programs were prepared and followed by same physician (OK). Therefore, 20 patients in one group had physical therapy after the first injection including TENS (transcutaneus electrical nerve stimulation), infrared ray and streching exercises for fifteen sessions applied by a physiotherapist. The follow up of patients was done at 3, 12 and 52 weeks after the first injection.

Statistical analysis was done by using SPSS v.15.0 for Windows software (SPSS, Chicago, IL, USA). Continuous variables are expressed as means or medians with standard deviations or minimum-maximum values. Categorical variables are presented as percentage frequencies. The Kolmogorov-Smirnov test was used to confirm that the data were within the ranges of normal distribution. We used Student's t-test for normally distributed data or Mann Whitney $U$ test for data that were not normally distributed. A p-value of 0.05 or less was chosen to reflect statistical significance. The comparison of repeated measures within groups was performed with the Friedman test. Paired comparisons for groups that were significant according to the Friedman test were compared using the Wilcoxon test with a value of $p<0.008$ considered to be significant by Bonferroni correction.

\section{Results}

The demographic data of the two groups are presented in Table 1.

\begin{tabular}{|lrccc|}
\hline \multicolumn{4}{|c|}{ Table 1. The demographic data of the groups } \\
\hline & Total & $\begin{array}{c}\text { Treatment } \\
\mathbf{1}\end{array}$ & Treatment $\mathbf{2}$ & $\begin{array}{c}\text { p } \\
\text { value }\end{array}$ \\
\hline Age & $51.9 \pm 15.0$ & $55.4 \pm 13.2$ & $48.4 \pm 16.2$ & 0.148 \\
\hline Gender & & & \\
\hline Female & $29(72.5)$ & $17(85)$ & $12(60)$ & 0.077 \\
\hline Male & $11(27.5)$ & $3(15)$ & $8(40)$ & \\
\hline$*: p<0.01$ (significant) & & & \\
\hline
\end{tabular}

There was no significant difference in the continuance of symptoms, pretreatment medication or baseline VAS, ODI and SF-36 between the groups in Table 2.

All patients in both groups had significant reduction in pain. At 52 weeks assessment, there was no statistically significant difference in pain relief between two groups. The pain and disability scores significantly improved from the baseline assessment in both groups at the 3rd, 12th and 52nd-week follow-up. There was no significant difference between the groups. The comparison of repeated measurements between the two groups is given in Table 3.

The comparison of differences between repeated measure- 
ments is presented in Table 4.

The comparison of SF-36 scores between groups is given in Table 5. No medical complication was seen during the follow-up period.

\begin{tabular}{lccc}
\hline \multicolumn{4}{c}{ Table 2. Comparison of VAS and ODI between two groups } \\
\hline & Treatment 1 & Treatment 2 & P value \\
\hline VAS 1 & $8.0 \pm 1.5$ & $7.7 \pm 1.7$ & 0.629 \\
\hline VAS 3 & $6.4 \pm 1.5$ & $5.8 \pm 2.1$ & 0.348 \\
\hline VAS 12 & $4.1 \pm 1.7$ & $3.6 \pm 2.1$ & 0.417 \\
\hline VAS 52 & $2.0 \pm 0.9$ & $1.6 \pm 0.9$ & 0.192 \\
\hline ODI 1 & $32.3 \pm 8.1$ & $28.0 \pm 7.1$ & 0.082 \\
\hline ODI 3 & $25.7 \pm 9.6$ & $22.1 \pm 6.7$ & 0.175 \\
\hline ODI 12 & $19.2 \pm 13.9$ & $15.5 \pm 9.1$ & 0.393 \\
\hline $\begin{array}{l}\text { ODI } 52 \\
\text { VAS: Visual Analog Scale (VAS) } 10 \mathrm{~cm} \\
\text { ODI: Oswestry Disability Index } \\
\text { Data are expressed as mean } \pm \text { SD, } p<0.01 \text { (significant) }\end{array}$ & & \\
\hline
\end{tabular}

Table 3. Comparison of repeated measurements

\begin{tabular}{|c|c|c|}
\hline & Group 1 & Group 2 \\
\hline \multicolumn{3}{|l|}{ VAS } \\
\hline TO & $8.0 \pm 1.5$ & $7.7 \pm 1.7$ \\
\hline T3 & $6.4 \pm 1.5^{a}$ & $5.8 \pm 2.1^{a}$ \\
\hline T12 & $4.1 \pm 1.7^{\mathrm{a}, \mathrm{b}}$ & $3.6 \pm 2.1^{a, b}$ \\
\hline T52 & $2.0 \pm 0.9 \mathrm{a}, \mathrm{b}, \mathrm{c}$ & $1.6 \pm 0.9^{a, b, c}$ \\
\hline \multicolumn{3}{|l|}{ ODI } \\
\hline T0 & $32.3 \pm 8.1$ & $23.6 \pm 12.4$ \\
\hline T3 & $25.7 \pm 9.6^{a}$ & $23.7 \pm 12.4^{a}$ \\
\hline T12 & $19.2 \pm 13.9^{a, b}$ & $27.6 \pm 15.3^{a, b}$ \\
\hline T52 & $9.3 \pm 6.8^{a, b, c}$ & $6.9 \pm 8.5^{a, b, c}$ \\
\hline \multicolumn{3}{|c|}{$\begin{array}{l}\text { VAS: Visual Analog Scale (VAS) } 10 \mathrm{~cm} \\
\text { ODI: Oswestry Disability Index } \\
\text { Data are expressed as mean } \pm \text { SD, T0: before injection, T3: } 3^{\text {td }} \text { week, T12: } 12^{\text {th }} \text { week, T52: } \\
52^{\text {nd }} \text { week, a: significant difference compared to TO assessment within group, b: significant } \\
\text { difference compared to T3 assessment within group, c: significant difference compared to } \\
\text { T12 assessment within group }\end{array}$} \\
\hline
\end{tabular}

Table 4. Comparison of differences between repeated measurements

\begin{tabular}{|c|c|c|c|c|}
\hline & Treatment 1 & Treatment 2 & $95 \% \mathrm{Cl}$ & $p$ value \\
\hline \multicolumn{5}{|c|}{ VAS Difference $(\Delta)$} \\
\hline$\Delta 0-3$ & $1.6 \pm 1.2$ & $1.8 \pm 1.2$ & $-1.0-0.6$ & 0.607 \\
\hline$\Delta 0-12$ & $4.0 \pm 1.4$ & $4.0 \pm 1.4$ & $-0.9-0.8$ & 0.707 \\
\hline$\Delta 0-52$ & $6.0 \pm 1.2$ & $6.0 \pm 1.4$ & $-0.8-0.8$ & 0.900 \\
\hline \multicolumn{5}{|c|}{ ODI Difference $(\Delta)$} \\
\hline$\Delta 0-3$ & $6.6 \pm 6.9$ & $5.9 \pm 5.8$ & $-3.4-4.7$ & 0.946 \\
\hline$\Delta 0-12$ & $13.1 \pm 13.9$ & $12.5 \pm 10.6$ & $-7.3-8.4$ & 0.626 \\
\hline$\Delta 0-52$ & $23.0 \pm 8.7$ & $21.1 \pm 10.6$ & $-4.3-8.1$ & 0.597 \\
\hline
\end{tabular}

VAS: Visual Analog Scale (VAS) $10 \mathrm{~cm}$

ODI: Oswestry Disability Index

Data are expressed as mean \pm SD. Cl: confidence interval, $p<0.01$ (significant)

Table 5. Comparison of SF-36 scores between groups

Treatment 1 Treatment 2 p value

PF 1

PF 3

PF 12

PF 52

RP 1

RP 3

RP 12

RP 52

RE 1

RE 3

RE 12

RE 52

EW 1

EW 3

EW 12

EW 52

EF 1

EF 3

EF 12

EF 52

SF 1

SF 3

SF 12

SF 52

BP 1

BP 3

BP 12

BP 52

$\mathrm{GH} 1$

$\mathrm{GH} 3$

$\mathrm{GH} 12$

$\mathrm{GH} 52$

SF-36: Short Form-36

PF: Physical functioning

RP: Role limitations due to physical health problems

RE: Role limitations due to personal or emotional problems

EW: Emotional well-being

EF: Energy/ fatigue

SF: Social functioning

BP: Bodily pain

$\mathrm{GH}:$ General health perceptions

Data are expressed as mean $\pm S D, p<0.01$ (significant)

\section{Discussion}

According to the literature including meta-analysis and randomized controlled trials prolotherapy is an effective treatment way in mild to moderate symptomatic knee osteoarthritis and overuse tendinopathy. But 10-20\% dextrose prolotherapy treatments in low back pain has confusing evidence about its efficacy $(2,3)$. According to a recent randomized double-blind 
controlled trial, $5 \%$ dextrose prolotherapy supplied notable relief for radicular low back pain and its effects endured as long as 1 year (6) Smigel et al. showed the analgesic and neurogenic influence of $10 \mathrm{~mL}$ volume $5 \%$ dextrose on pain with epidural injections and subcutaneous injections. It has been reported that $5 \%$ dextrose has an effect of reducing hyperalgesia, allodynia and neurogenic pain. They showed for the first time that $5 \%$ dextrose injected in the caudal space can cause a pain-specific neurogenic effect at the dorsal root level (6).

In another study it was shown that $5 \%-10 \%$ dextrose has the influence of changing the effectiveness of epidural anesthesia and has not been associated with complications (7). Dextrose injection is used to manage the pain and improve the physical functions in shoulder disorders including rotator cuff tendinopathy, osteoarthritis, lateral epicondylosis, Osgood-Schlatter disease, sacroiliac joint dysfunction, temporomandibular dysfunctions, non-specific, non-surgical low back pain, achilles tendinopathies, adductor and plantar fasciitis (8-17). Dextrose is also thought to reduce pain through a sensorineural pathway (18). But still in some clinical conditions information about the effectiveness of prolotherapy is insufficient. For example for temporomandibular dysfunction although the results are promising but not sufficient for suggestion (8). Findings for non-specific low back pain in the literature is confusing because control and patient groups received different treatment protocols, there are no standart protocols about indications, solution concentrations and treatment protocols. There is not enough proof to advise prolotherapy for non-specific low back pain. However, other randomized controlled trials have promising results reporting advances for pain and disability in patients with chronic, moderate-to-severe low back pain (10). There is contradictory proof about the effect of prolotherapy injections for patients with chronic low-back pain. According to some of the previous studies prolotherapy when used alone is not an efficient treatment for chronic low-back pain, when combined with other treatments such as exercise or spinal manipulation prolotherapy was found useful for chronic low-back pain and disability. In fact, the result of such studies is confusing because of the clinical heterogeneousness amongst studies and the differences of the other combined supporting treatments. According to another systematic review, there was no proof that prolotherapy injections were more useful than other injections. In this review the outcome measures were pain and disability levels and two studies showed important differences between the treatment and control groups in terms of pain reduction or disability. The results of the studies could not be combined because of the heterogeneity of co-interventions. In a randomized controlled trial subjects with chronic low back pain, were randomized to one of four treatment groups: dextrose and normal activity, dextrose and physical therapy, saline injections and physical therapy, or saline injections and normal activity . In one year, subjects in all groups described improvement in disability scores and pain without significant differences between groups (19). In another randomized controlled trial patients with chronic low back pain, buttock or leg pain were treated with $10 \mathrm{~mL}$ of dextrose injected in the caudal epidural space, and this treatment was compared with injection of $10 \mathrm{~mL}$ of normal saline. The results of this study showed a short-term analgesic effect of dextrose for chronic low back pain with radiation to buttock or leg (20) In a prospective case series with patients having degenerative discogenic leg pain who had failed other treatments fluoroscopically-guided injections to the related disc space with $25 \%$ dextrose with bupivacaine, showed an import- ant prolonged effect in pain score at 2 nd and 18th months. In fact there is a need for future randomized controlled trials about clinical indications of low back pain (21).

To our knowledge, there is no study in the literature about the effect of $5 \%$ dextrose prolotherapy for radicular low back pain. Our study results suggest that prolotherapy is an effective treatment method for radicular low back pain and have advantages over other techniques as this method is more effective, safe and cheap.

Nonetheless, several limitations should be recognized for the present study. Small sample size is major limitation which might have precluded generalization of the results. Other limitation is the lack of control group and its' retrospective design However, the findings of the present study provide long-term effectiveness of prolotherapy.

As a result; patients with radicular low back pain caused by lombar disc herniation can be treated effectively by using $5 \%$ dextrose prolotheraphy. Since the rehabilitation clinics are so busy and patients have to get an appointment sometimes longing for months for receiving physical medicine program, we can prefer only prolotheraphy as an effective treatment method for radicular low back pain due to lombar disc herniation.

Acknowledgements: OK collected the data, reviewed the study results and contributed the writing of the manuscript. AO directed the study. IS designed the study, performed the injections YD analyzed the study results and OK contributed the revision of manuscript. No financial support was received from any source for this work.

Conflict of interest: The authors declared no conflict of interest with respect to this manuscript.

\section{References}

1. Fullerton BD. Prolotherapy for the thoracolumbar myofascial system. Phys Med Rehabil Clin N Am. 2018;29(1):125-138.

2. Dagenais S, Yelland MJ, Del Mar C, Schoene ML. Prolotherapy injections for chronic low- back pain. [published online April 18 2007]. Cochrane Database Syst Rev doi: 10.1002/14651858.CD004059.pub3

3. Rabago D, Nourani B. Prolotherapy for osteoarthritis and tendinopathy: A descriptive review. Curr Rheumatol Rep. 2017;19(6):34-39.

4. Solmaz I, Orscelik A. Features and clinical effectiveness of the regenerative injection treatments: Prolotherapy and platelet-rich plasma for musculoskeletal pain management. In: From conventional to innovative approaches for pain treatment. 1 st ed. Intech Open Publishing Co; 2019.

5. Guran S, Coban ZD, Karasimav O, et al. Dextrose solution used for prolotherapy decreases cell viability and increases gene expressions of angiogenic and apopitotic factors. Gulhane Medical Journal. 2018;60(2):42-46.

6. Maniquis-Smigel L, Dean Reeves K, Jeffrey Rosen H, et al. Short term analgesic effects of $5 \%$ dextrose epidural injections for chronic low back pain: A randomized controlled trial. Anesth Pain Med. 2016;7(1):e42550.

7. Liu S, Pollock JE, Mulroy MF, Allen HW, Neal JM, Carpenter RL. Comparison of $5 \%$ with dextrose, $1.5 \%$ with 
dextrose, and $1.5 \%$ dextrose-free lidocaine solutions for spinal anesthesia in human volunteers. Anesth Analg. 1995;81(4):697-702.

8. Reeves KD, Sit RW. Dextrose prolotherapy: A narrative review of basic science, C-clinical research, and best treatment recommendations. Phys Med Rehabil Clin N Am. 2016;27(4):783-823.

9. Hackett GS. Prolotherapy in whiplash and low back pain. Journal of Postgraduate Medicine. 1960;27(1):214-219.

10. Rabago D, Slattengren A, Zgierska A. Prolotherapy in primary care practice. Prim Care. 2010;37(1):65-80.

11. Bertrand H, Reeves KD, Bennett CJ, Bicknell S, Cheng $\mathrm{AL}$. Dextrose prolotherapy versus control injections in painful rotator cuff tendinopathy. Arch Phys Med Rehabil. 2016;97(1):17-25.

12. Rabago D, Patterson JJ, Mundt M, et al. Dextrose prolotherapy for knee osteoarthritis: a randomized controlled trial. Ann Fam Med.2013;11(3):229-237.

13. Topol GA, Podesta LA, Reeves KD, Raya MF, Fullerton BD, Yeh HW. Hyperosmolar dextrose injection for recalcitrant Osgood-Schlatter disease. Pediatrics. 2011;128(5):1121-1128.

14. Jahangiri A, Moghaddam FR, Najafi S. Hypertonic dextrose versus corticosteroid Local injection for the treatment of osteoarthritis in the first carpometacarpal joint: a double-blind randomized clinical trial. J Orthop Sci. 2014;19(5):737-743.

15. Scarpone M, Rabago DP, Zgierska A, Arbogast G, Snell E. The efficacy of prolotherapy for lateral epicondylosis: a pilot study. Clin J Sport Med.2008;18(3):248-254.

16. Rabago D, Lee KS, Ryan M, et al. Hypertonic dextrose and morrhuate sodium injections (prolotherapy) for lateral epicondylosis (tennis elbow): results of a singleblind, pilot-level, randomized controlled trial. Am J Phys Med Rehabil. 2013;92(7):587-596.

17. Kim WM, Lee HG, Jeong CW, Kim CM, Yoon MH. A randomized controlled trial of intra-articular prolotherapy versus steroid injection for sacroiliac joint pain. J Altern Complement Med. 2010;16(12):1285-1290.

18. Malek N, Pajak A, Kolosowska N, Kucharczyk M, Starowicz K. The importance of TRPV1-sensitisation factors for the development of neuropathic pain. Mol Cell Neurosci. 2015;65(3):1-10.

19. Yelland MJ, Del Mar C, Pirozzo S, Schoene ML. Prolotherapy injections for chronic low back pain: A systematic review. Spine 2004;29(19):2126-2133.

20. Ostelo RW, Deyo RA, Stratford P, et al. Interpreting change scores for pain and functional status in low back pain: towards international consensus regarding minimal important change. Spine (Phila $\mathrm{Pa}$ 1976). 2008;33(1):90-94.

21. Miller MR, Mathews RS, Reeves KD. Treatment of painful advanced internal lumbar disc derangement with intradiscal injection of hypertonic dextrose. Pain Physician. 2006;9(2):115-121. 